\title{
A ATUAÇÃO DO FARMACÊUTICO NO COMBATE A AUTOMEDICAÇÃO
}

\author{
Anne Caroline Santiago Guedes ${ }^{1}$ \\ Leonardo Guimarães de Andrade ${ }^{2}$
}

RESUMO: A automedicação é uma prática difundida não apenas no Brasil, como em outros países. Em uma sociedade com hábitos de consumo de medicamentos as políticas nacionais acabam por promover a disponibilização de medicamentos sem diagnóstico ou prescrição por profissionais habilitados. O trabalho tem o objetivo de investigar o papel do farmacêutico no combate a prática de auto medicalização. Automedicação é a administração de medicamentos sem orientação ou prescrição médica. $O$ hábito de automedicar-se pode provocar danos à saúde e até mascarar sintomas de doenças mais graves. O farmacêutico é o profissional que conhece e é responsável por medicamento, portanto, deve fornecer informações. $O$ presente estudo caracteriza-se ainda como uma pesquisa bibliográfica, que faz um levantamento do conhecimento atual veiculado na literatura sobre administração que podem trazer contribuições para o tema pesquisado. Usou-se autores como Conselho Federal de Farmácia (2011), Arrais (1997), Naves et al. (2010), entre outros.

Palavras-Chave: Farmácia. Automedicação. Prescrição.

ABSTRACT: Self-medication is a widespread practice not only in Brazil, but also in other countries. In a society with drug consumption habits, national policies end up promoting the availability of drugs without diagnosis or prescription by qualified professionals. The work aims to investigate the role of the pharmacist in combating the practice of selfmedicalization. Self-medication is the administration of medication without medical advice or prescription. The habit of self-medicating can cause damage to health and even mask symptoms of more serious illnesses. The pharmacist is the professional who knows and is responsible for the medication and, therefore, must provide information. This study is also characterized as bibliographical research, which surveys the current knowledge published in the literature on administration that can contribute to the researched topic, which surveys current knowledge in the literature and uses authors such as the Federal Council of Pharmacy (2011), Arrais (1997), Naves et al. (2010), among others.

Key words: Pharmacy. Self-medication. Prescription.

\footnotetext{
I Graduação em Farmácia. UNIG. E-maill.: annesantiago2ı@gmail.com

${ }^{2}$ Mestre em Ciências do Meio Ambiente Universidade Veiga de Almeida. Mestre em Doenças Parasita Universidad Autônoma de Assuncion. Formação Graduação em Enfermagem . Formação Graduação em Odontologia.E-mail: Leonard.gui@homail.com
} 


\section{INTRODUÇÃO}

A automedicação é o ato de tomar medicamentos por contra própria sem uma prescrição médica. Muitas vezes proporcionando um meio mais fácil e de rápida solução para o alivio de sintomas correntes e que pode não levar a ideia da importância de procurar um serviço médico. Ela também é definida como a prática de uso de medicamentos que não precisam de receita médica para tratar pequenos males, como resfriados, enxaqueca, azia etc. E o risco está correlacionado com o grau de informação sobre os medicamentos e com a necessidade do próprio medicamento.

Além disso mais um motivo que faz o paciente automedicar-se é a objeção de consegui chegar à saúde pública.

Considera-se um problema de saúde pública, uma vez que põe em risco a condição de saúde dos usuários, podendo gerar não só a deturpação de sinais e sintomas de diferentes doenças, como, dificulta assim o diagnóstico e pode prejudicar o tratamento.

A prática acaba por ser reforçada porque muitos medicamentos podem ser

adquiridos sem prescrição médica, ainda assim, o uso destes produtos não deve ser feito de maneira indiscriminada, já que para a sua ingestão é necessária uma dose certa respeitando ainda o tempo para que seja eficaz.

Tendo em vista como profissional da saúde, o farmacêutico tem papel fundamental na assistência, no aconselhamento e orientação para garantir a segurança do cliente na dispensação e no manejo dos fármacos. Uma vez que a sociedade tem a farmácia como escolha principal para cuidados médicos, necessitando de comprovação de que a utilização de forma errada de fármacos pode trazer danos graves a saúde (ROCHA,2OII).

\section{JUSTIFICATIVA}

O Trabalho busca investigar o uso indiscriminado de medicamentos e o papel do profissional farmacêutico no combate a prática de automedicação, desse modo, pode-se dizer que se justifica a partir da finalidade de contribuir para o aprimoramento acadêmico e pessoal, assim como visa também acrescenta à comunidade acadêmica com o fomento da discussão do tema e de somar com novas perspectivas a partir dos autores citados. Além 
disso, traz contribuições para o social, já que é um tema que levanta questões de saúde e que afetam a sociedade no geral, uma vez que está é uma prática comum no dia a dia.

\title{
2. OBJETIVOS
}

\section{I Geral}

O trabalho tem o objetivo de investigar o papel do farmacêutico no combate a prática de auto medicalização.

\subsection{Específicos}

- Investigar o papel do farmacêutico no combate a automedicação;

- Entender o hábito da automedicação;

- Discutir ações que podem coibir a automedicação.

\section{METODOLOGIA}

O presente estudo caracteriza-se ainda como uma pesquisa bibliográfica, que faz um levantamento do conhecimento atual veiculado na literatura que podem trazer contribuições para o tema pesquisado.

\begin{abstract}
A pesquisa bibliográfica é feita a partir do levantamento de referências teóricas já analisadas, e publicadas por meios escritos e eletrônicos, como livros, artigos científicos, páginas de web sites. Qualquer trabalho científico inicia-se com uma pesquisa bibliográfica, que permite ao pesquisador conhecer o que já se estudou sobre o assunto (FONSECA, 2002, p.32).
\end{abstract}

O trabalho se apoia em livros, artigos, revistas eletrônicas, entre outros materiais científicos e está baseado em autores da área e serão levantados livros e artigos científicos, na base de dados Scielo, repositórios online de universidades, revistas científicas e bibliotecas físicas e virtuais.

\section{DESENVOLVIMENTO}

Segundo Fernandes et al., (2020), os medicamentos estão inseridos em muitos contextos da atenção à saúde, pois são a forma mais comum disponível de terapia na sociedade contemporânea. Entende-se que o seu uso é essencial e fundamental para a manutenção da saúde, e por essa razão, o seu acesso, assim como o modo que é utilizado e 
o descarte acabaram se tornando um problema de saúde pública, porque passou a ser feito em excesso.

O desenvolvimento de fármacos possibilitou transformações nas atividades de assistência à saúde e sendo assim, o medicamento é uma tecnologia difundida e muito utilizada. Contudo, com o advento do capitalismo e com o expressivo crescimento do consumo desses itens farmacêuticos em conjunto com o modelo de atenção à saúde focado no tratamento de doenças, fez com que o uso de medicamentos se tornasse cada vez maior tornando-se em muitos casos, abusivo e colocando a população diante de riscos relacionados ao seu uso.

O setor privado é o responsável por fornecer fármacos e medicamentos no Brasil, enquanto as farmácias são responsáveis pela comercialização de medicamentos e distribuição para a população em geral, o que coloca em maioria nas mãos de leigos, ou seja, de proprietários e balconistas (NAVES et al., 2010).

Em uma sociedade com hábitos de consumo de medicamentos pode ser positiva a criação de políticas nacionais que promovem a regulamentação do suprimento e a disponibilização de medicamentos essenciais, pressupondo o acesso ao diagnóstico e prescrição por profissionais habilitados. Porém, por outro lado, o consumo pode ser influenciado negativamente pelo acesso sem barreiras e pela promoção e publicidade de medicamentos, que em sua maioria, estimulam a utilização desnecessária dos mesmos (NAVES et al., 2010).

\section{Figura I}

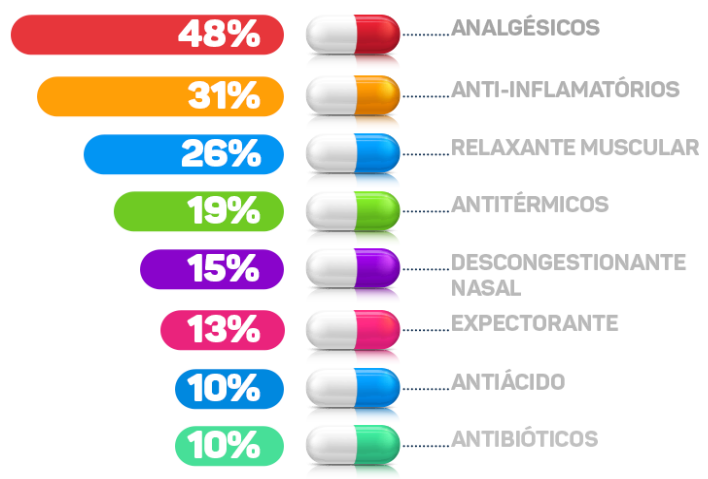

Fonte: https://ictq.com.br/pesquisa-do-ictq/871-pesquisa-automedicacao-no-brasil-2018 
Segundo o Conselho Federal de Farmácia (20II) os farmacêuticos são profissionais da área da saúde, e tem uma função na sociedade, uma vez que é fundamental o trabalho do profissional na manipulação de fármacos e medicamentos e isso requer um saber específico já que trazem consequências ao organismo humano e animal. Desse modo, o trabalho de um farmacêutico vai desde indicar, aconselhar, deve ser o também de atuar na prevenção da automedicação. Podemos dizer assim, que este é um profissional multicomponente na sociedade. Além disso, ainda segundo o Conselho Federal de Farmácia (20II) este profissional é responsável por um “[...] conjunto de ações e serviços com vistas a assegurar a assistência terapêutica integral, a promoção, a proteção e a recuperação da saúde nos estabelecimentos públicos ou privados, desempenhados pelo farmacêutico ou sob sua supervisão".

Podemos dizer que faz parte das obrigações do farmacêutico a ação de orientar com o objetivo de combater a automedicação. Desse modo, deve se preparar para atuar na atenção farmacêutica como estratégia para diminuir o uso indiscriminado de medicamentos. Segundo Merhy (1993), é possível afirmar que o papel social deste profissional na promoção de saúde inclui ações relacionadas a medicamentos

Deve ser especialista em informações sobre medicamentos, deve orientar o paciente quanto ao uso dos medicamentos, deve acompanhar os resultados do tratamento ou ainda orientar ao paciente para que este não interrompa o tratamento advertindo-o em relação aos danos que o mesmo pode causar a saúde do paciente; etc. (MERHY, 1993, p. 46).

Segundo a Lei no 5.991 de 19 de dezembro de 19973 do Conselho Federal de Farmácia (20II), determina que o profissional de farmácia é o responsável técnico pelas farmácias e além disso são incumbidos de programar, comprar, distribuir, dispensa, garantir a qualidade dos produtos e serviços, acompanhamento e avaliação de sua utilização, na perspectiva de obtenção de resultados concretos e de melhoria da qualidade de vida da população. Por essa razão, o acesso, aos medicamentos, tem uma finalidade específica, na dosagem correta, pelo tempo necessário, com a garantia de qualidade e a informação suficiente para o uso adequado, tendo como objetivo a solução das questões de saúde. Desse modo, o acesso a essa medicação deve ser feita de maneira racional e segura, e a partir da articulação de ações inseridas na assistência farmacêutica e um conjunto de ações de atenção à saúde. 
Das funções do profissional de farmácia, está incluída a prática de dispensação que diz respeito ao atendimento farmacêutico de orientar o paciente sempre que que é lhe apresentado uma receita médica. Segundo o Ministério da Saúde no Relatório de Políticas Nacional de Medicamentos (2001), a dispensação é ação de proporcionar medicamentos a pacientes, como resposta à uma receita, além disso, o farmacêutico deve informar e orienta o paciente sobre o uso adequado do medicamento, de forma que essas informações e orientações sobre a importância do respeito a dosagem, a influência da alimentação no resultado, além da interação com outros medicamentos, reações adversas e ainda sobre a conservação dos fármacos. Dessa maneira, podemos entender que como boas práticas, existe um conjunto de normas que estabelecem regras que garantem um bom trabalho.

Para obter alívio de incômodos, muitas vezes, a partir do aparecimento de sintomas, principalmente os mais comuns como os que surgem a partir de viroses, os indivíduos se veem impulsionados a fazer uso de medicamentos para gripe, febre, dor de garganta, etc. ou ainda a receber orientação de amigos, farmacêutico amigo, com objetivo de conseguir uma solução medicamentosa. Além disso, a mídia através dos seus meios de comunicação e propaganda acabam por estimular a prática de automedicação (AUTOMEDICAÇÃO, 200I).

\section{Figura 2}

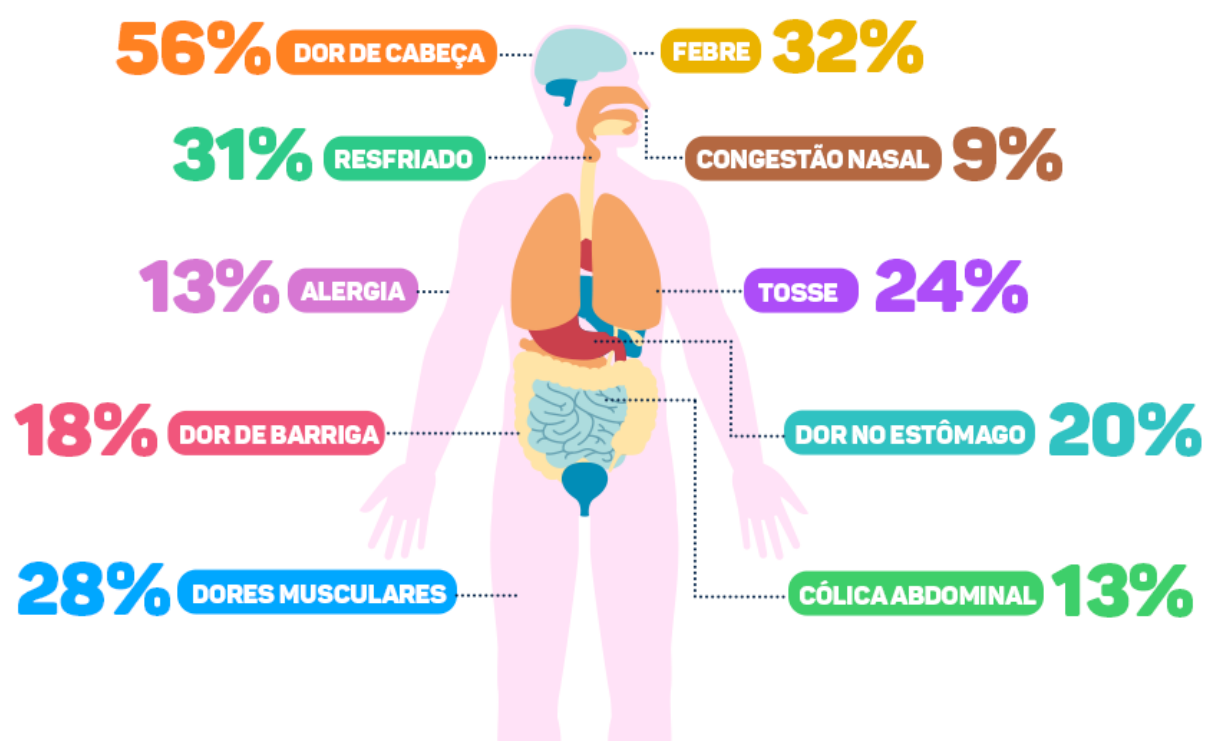


Fonte: https://ictq.com.br/pesquisa-do-ictq/871-pesquisa-automedicacao-no-brasil-2018

Segundo Zubioli (200o, p. 45) a inclusão do farmacêutico no processo de automedicação responsável começa com a percepção do problema de saúde pelo usuário, dessa maneira, é preciso que o profissional tenha a noção da competência e dos limites de sua intervenção no processo saúde/doença para que possa então assumir uma postura que esteja condizente com as determinações do seu conselho em relação ao que deve ou não ser feito, e ser ainda capaz de avaliar a situação do usuário, conduzindo-o quando preciso a uma consulta médica.

A prática de automedicação diz respeito segundo Afonso (2006) do processo em que o usuário decide se automedicar sem prévio conhecimento sobre a medicação e se ela pode lhe trazer benefícios ou não para determinado mal-estar, colocando assim, sua saúde em risco.

Pode-se entender como uma prática que coloca a saúde do usuário em risco, uma vez que pode acarretar problemas à saúde, já que iniciar um tratamento medicamentoso, exige uma investigação competente a respeito do processo de adoecimento e deve ser feito por um profissional de saúde. Esta prática traz vantagens e desvantagens, segundo Naves et al. (2010) e entre as desvantagens, está a possibilidade de agravamento de problemas de saúde, já que em muitos casos não há orientação e o tratamento correto. "O problema é universal, antigo e de grandes proporções. A automedicação pode ser considerada uma forma de não adesão às orientações médicas e de saúde" (AUTOMEDICAÇÃO, 20oI, p. 270).

Para Arrais (1997) a automedicação feita de maneira inadequada, assim como um erro de prescrição, pode trazer consequência e efeitos indesejáveis, doenças iatrogênicas e mascaramento de outras doenças evolutivas, sendo, portanto, um problema a ser combatido. A automedicação é um problema de saúde pública pelos riscos que essa prática coloca a condição de saúde do indivíduo em risco (TRUTA et. al., 2010).

Além disso, traz riscos como intoxicação por uso inadequado de medicamento que são muitas vezes induzidas pela ingestão feita pelas diversas propagandas publicitárias persuasivas que prometem alívio rápido (CARVALHO; GARCIA, 2003). A má administração de medicamento também pode ocasionar problemas renais e falência do 
fígado, órgão responsável pelo metabolismo de substâncias tóxicas (MARQUES; OLIVEIRA; MENEZES, 2013).

A prática de consumo de medicamentos (automedicação) feito de forma indiscriminada deve ser combatida, uma vez que está ligada a compra e venda de medicamentos sem receita. Isso deve ser feito por questões de saúde pública (BORTOLON, 2007).

Para Naves et al. (2010) as farmácias aparecem a solução rápida e de fácil acesso, mas não fornecem junto aos medicamentos orientações educativas ou preventivas de qualidade, nem muito menos acompanhamento. Desse modo, a partir da lógica de mercado alimentados pela venda de medicamentos e pela concepção reducionista de saúde e doença, transformou a saúde em mercadoria e os medicamentos e as tecnologias associadas ganharam crescente autonomia, diminuindo a importância das pessoas no processo de cura.

Ainda segundo os autores a prática da automedicação está inserida no cotidiano da população, isso porque oferece autonomia no cuidado e no restabelecimento de um estado de saúde desejado através do uso de recursos terapêuticos convenientes a cada um (NAVES et al., 2010).

\section{CONSIDERAÇÕES FINAIS}

A automedicação é uma prática que já faz parte dos costumes e hábitos da população e vem sendo uma atitude passada pelas gerações. Pode-se dizer que ela parte de uma deficiência do Sistema Único de Saúde por não oferecer um serviço de farmácias de qualidade e nem de prestação de serviço de saúde, que acaba por forçar o usuário a se automedicar por não ter assistência segura. Isso tem como resultado em farmácias comerciais vendendo medicamentos sem receita médica de maneira indiscriminada.

Por essa razão o trabalho do farmacêutico se faz indispensável, partindo de um trabalho ético e seguindo as normas e aquilo que é permitido a ele como: programar, comprar, distribuir, dispensa, garantir a qualidade dos produtos e serviços, acompanhamento e avaliação de sua utilização, além a dispersão, que é a prática de entregar ao usuário o medicamento indicado a partir de uma receita. 
Desse modo, podemos entender que a função do farmacêutico na ação de coibir a automedicação é a de informar a população dos perigos da prática, uma vez que muitos medicamentos não necessitam de indicação, além de negar o acesso através da venda de medicamentos que exigem receita médica.

\section{CONCLUSÃO}

O Farmacêutico é o profissional capacitado que adquiri o conhecimento e aspectos dos medicamentos e, por esse motivo, ele é quem pode fornecer uma informação segura às pessoas que o procuram, seja nas farmácias, drogarias. Promove uma qualidade de vida melhor para os clientes dificultando a automedicação, que pode causar riscos com uso inadequado de medicamentos levando danos gravíssimos saúde do consumidor.

\section{REFERÊNCIAS}

AFONSO JR. SUS para valer: com saúde fiscal e federatividade. Belo Horizonte: Seminário, 2006.

AUTOMEDICAÇÃO. Revista da Associação Médica Brasileira [online]. 20oI, v. 47, n. 4 [Acessado I9 Setembro 2021], pp. 269-270. Disponível em: <https://doi.org/ro.1590/Soro442302001000400001>. Epub 23 Jan 2002. ISSN 1806-9282. https://doi.org/10.1590/Soro442302001000400001 .

ARRAIS, P.S. D. Perfil da automedicação no Brasil. Rev. Saúde Pública, I997.

BORNOLON, P.C. Automedicação versus indicação farmacêutica: O profissional da farmácia da atenção primária à saúde do idoso. Rev. APS, v.ı, № 2, p. 200-209. Julho/Dezembro, 2007.

BRASIL. Ministério da Saúde. Política Nacional de Medicamentos 200r. Relatório da Política Nacional de Medicamentos. Brasília: Ministério da Saúde, 20oı. 
CONSELHO FEDERAL DE FARMÁCIA. Atuação do Farmacêutico. Brasília, 20 II.

FERNANDES, Mayra Rodrigues et al. Armazenamento e descarte dos medicamentos vencidos em farmácias caseiras: problemas emergentes para a saúde pública. Einstein (São Paulo), São Paulo, v. I8. Disponível em: 〈http://www.scielo.br/scielo.php?script=sci_arttext\&pid=SI67945082020000100238\&lng=en $\& \mathrm{nrm}=\mathrm{iso}>$. Acesso em $\mathrm{I} 4$ de setembro de 202I.

FONSECA, J. J. S. Metodologia da pesquisa científica. Fortaleza: UEC, 2002.

MERHY, Emerson E. Saúde Pública. Rio de Janeiro abril/jun. 1993.

NAVES, Janeth de Oliveira Silva et al. Automedicação: uma abordagem qualitativa de suas motivações. Ciência \& Saúde Coletiva [online]. 2010, v. 15, suppl I [Acessado I9 Setembro 2021], pp. 1751-1762. Disponível em: 〈https://doi.org/10.1590/Si41381232010000700087>. Epub o8 Jul 2010. ISSN 1678-4561. https://doi.org/ro.1590/Si41381232010000700087 .

TRUTA CN, MAURÍCIO VAS, MAGALHÃES FC, SILVA GF, FERNANDES GV, QUEIROZ RML, ET AL. Prevalência e Características da Automedicação entre os Idosos: Revisão Bibliográfica. XV Encontro Latino-Americano de Iniciação Científica e XI Encontro Latino Americano de Pós-Graduação Universidade do Vale da Paraíba, 20 o.

ZUBIOLI, Arnaldo. O Farmacêutico e a automedicação responsável. Pharmacia Brasileira, 20oo. Disponível em: https://www.cff.org.br/sistemas/geral/revista/pdf/roo/6.pdf. Acesso: 17 de setembro de 2021.

Figura i e 2 encontradas respectivamente nas paginas 6 e 8. Pesquisa pelo ICTQ sobre Auto Medicação no Brasil (2018). Disponível em: https://ictq.com.br/pesquisa-doictq/87I-pesquisa-automedicacao-no-brasil-2018 
Revista Ibero- Americana de Humanidades, Ciências e Educação- REASE

open 2 access 